\title{
The Role of Functional Prion-Like Proteins in the Persistence of Memory
}

\author{
Kausik $\mathrm{Si}^{1,2}$ and Eric R. Kande $\mathrm{l}^{3,4,5,6}$ \\ ${ }^{1}$ Stowers Institute for Medical Research, Kansas City, Missouri 64113 \\ ${ }^{2}$ Department of Physiology, School of Medicine, University of Kansas Medical Center, Kansas City, \\ Kansas 66160 \\ ${ }^{3}$ Howard Hughes Medical Institute, Chevy Chase, Maryland 20815-6789 \\ ${ }^{4}$ Departments of Neuroscience and Psychiatry, College of Physicians and Surgeons of Columbia University, \\ New York, New York 10027 \\ ${ }^{5}$ Zuckerman Mind Brain Behavior Institute, New York State Psychiatric Institute, New York, New York 10032 \\ ${ }^{6}$ Kavli Institute for Brain Sciences, New York, New York 10032 \\ Correspondence: ksi@stowers.org
}

Prions are a self-templating amyloidogenic state of normal cellular proteins, such as prion protein (PrP). They have been identified as the pathogenic agents, contributing to a number of diseases of the nervous system. However, the discovery that the neuronal RNA-binding protein, cytoplasmic polyadenylation element-binding protein (CPEB), has a prion-like state that is involved in the stabilization of memory raised the possibility that prion-like proteins can serve normal physiological functions in the nervous system. Here, we review recent experimental evidence of prion-like properties of neuronal CPEB in various organisms and propose a model of how the prion-like state may stabilize memory.

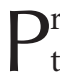
ions are proteinaceous infectious agents that were discovered in the 1980s by Stanley Prusiner while studying Creutzfeldt-Jakob disease (Prusiner 1982). Prusiner and colleagues showed them to be an amyloidogenic, self-perpetuating, forms of a normal cellular protein, termed prion protein or PrP. Prp in its self-perpetuating state kills cells. Prusiner and colleagues found that PrPs exist in at least two conformations: monomeric and aggregated (Fig. 1). The transition among these forms occurs spontaneously and only the aggregated conformation is pathogenic. Soon, PrPs were found to contribute to other neurodegenerative disorders in people, including kuru, transmissible spongiform encephalopathies, as well as bovine spongiform encephalopathy in cows (Prusiner 1994; Aguzzi and Weissmann 1998).

There is now a growing consensus that similar prion-like, self-templating mechanisms underlie a variety of neurodegenerative disorders, including amyotrophic lateral sclerosis, Alzheimer's disease, Parkinson's disease, and Huntington's disease (Polymenidou and Cleveland 2012).

Not all prions, however, appear to be disease causing. Fungal prions, for instance, are nontoxic, and some may even be beneficial to the

Editors: Eric R. Kandel, Yadin Dudai, and Mark R. Mayford

Additional Perspectives on Learning and Memory available at www.cshperspectives.org

Copyright (C) 2016 Cold Spring Harbor Laboratory Press; all rights reserved; doi: 10.1101/cshperspect.a021774

Cite this article as Cold Spring Harb Perspect Biol 2016;8:a021774 
K. Si and E.R. Kandel

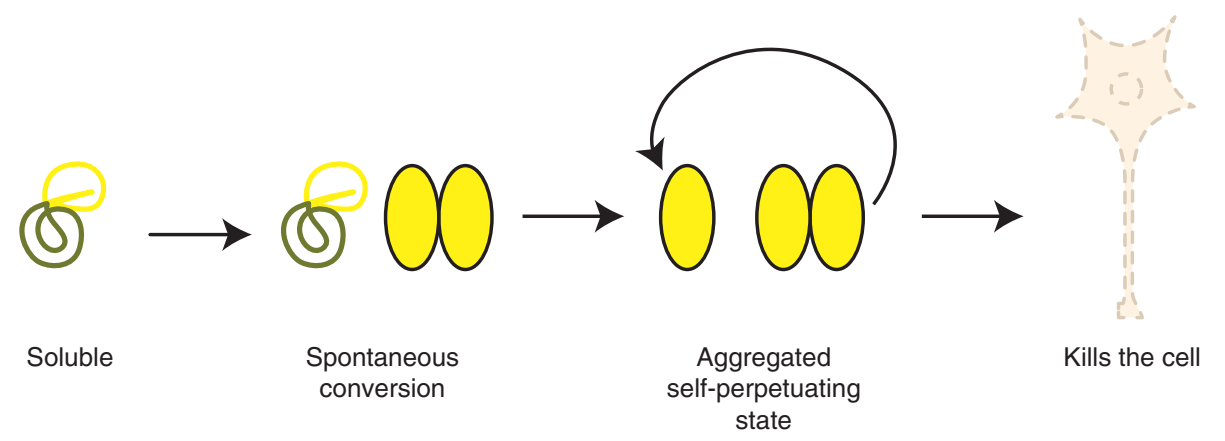

Figure 1. Pathogenic prions exist in two states (soluble and aggregated and self-perpetuating). The conversion from the soluble to the aggregated form is spontaneous and the aggregated, self-perpetuating form is often toxic and kills the cell.

cells that harbor them (Wickner 1994; Shorter and Lindquist 2005; Crow and Li 2011). In 2003, Si and Kandel serendipitously discovered a prion-like protein in multicellular eukaryotes-the nervous system of the marine snail Aplysia-whose aggregated and self-perpetuating form contributes to the maintenance of long-term changes in synaptic efficacy. This functional prion-like protein differs from pathogenic prions in two important ways: (1) The conversion to the prion-like state is regulated by a physiological signal, and (2) the aggregated form has an identified physiological function (Fig. 2). Recent identification of new functional prion-like proteins in various organisms, including human, supports the idea that non- pathogenic prions may perform a wide range of biologically meaningful roles (Coustou et al. 1997; Eaglestone et al. 1999; True and Lindquist 2000; Ishimaru et al. 2003; True et al. 2004; Hou et al. 2011; Jarosz et al. 2014).

In this review, we focus on functional prionlike proteins in the brain and specifically on the prion-like properties of the cytoplasmic polyadenylation element-binding protein (CPEB), and examine how the prion-like state can control protein synthesis at the synapse and, thereby, synaptic plasticity and long-lasting memory. We anticipate the studies of CPEB would also provide some generalizable concepts as to how prion-based protein switches in multicellular eukaryotes may work.

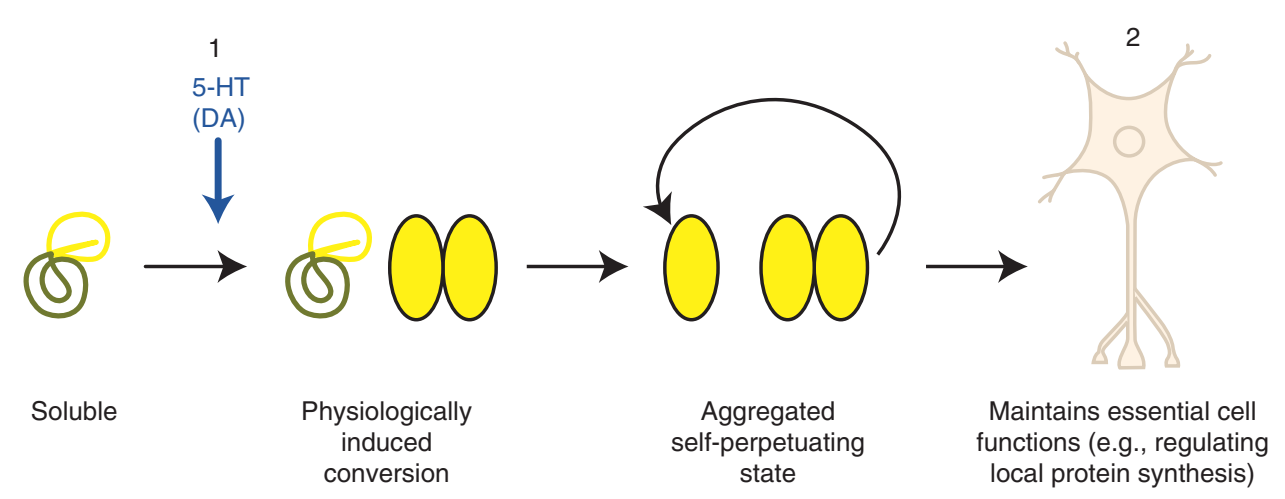

Figure 2. "Functional" prion: memory. "Functional" prions differ from conventional prions in two ways. First, the conversion is triggered by a physiological signal, and second, the aggregated, self-perpetrating forms have a physiological function. 5-HT, Serotonin; DA, dopamine. 


\section{A METASTABLE PROTEIN CONFORMATION SWITCH FOR SYNAPSE-SPECIFIC LOCAL PROTEIN SYNTHESIS}

Synapses Are Independent Units of Change

A single neuron can have up to $10^{3}$ synapses. These are the units of information storage for short-term memory. Because long-term memory storage requires gene expression, which takes place in the nucleus, one might expect longterm memory to involve cell-wide changes. To explore whether individual synapses can also serve as units for long-term memory lasting days and weeks, Martin and colleagues (Martin et al. 1997a; Casadio et al. 1999) performed experiments in which serotonin $(5-\mathrm{HT})$, the modulatory transmitter released during training for learned fear in Aplysia, was applied locally to one of the two branches of the bifurcating sensory neurons that innervate two separate motor neurons. These experiments, as well as parallel experiments by Frey and Morris in the rodent hippocampus (Frey and Morris 1997), show that individual synapses can be independently modified to give rise to long-term synaptic plasticity, which persists for $>24 \mathrm{~h}$ (Fig. 3). Thus, longterm facilitation (LTF) and its associated synaptic changes are synapse-specific. Moreover, this synapse specificity requires the action of the transcription factor cyclic adenosine-3-monophosphate (cAMP)-response element-binding protein 1 (CREB-1). Blocking CREB-1 expression blocks the synapse-specific facilitation. These findings imply that signals are sent not

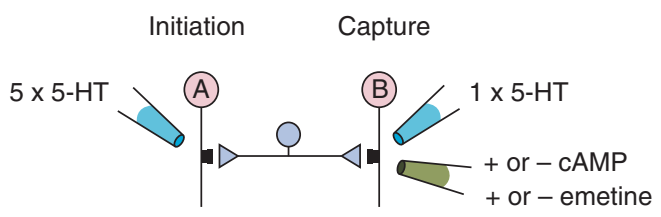

Synaptic capture + or - cAMP: cell B

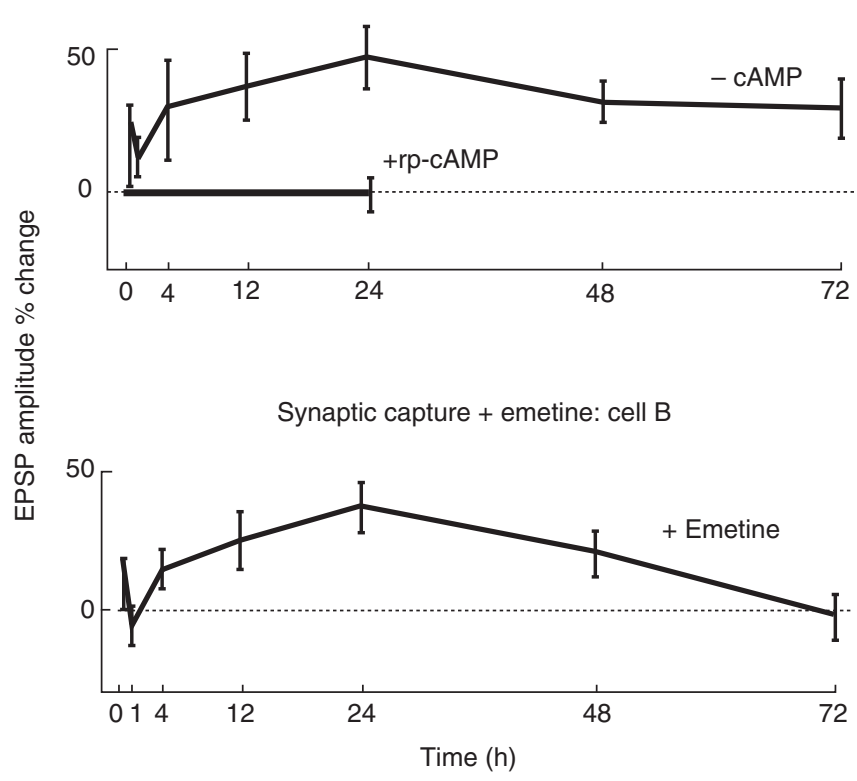

Figure 3. There are two molecular components to the "synaptic" mark (Martin et al. 1997a,b). (Top) Activation of the cyclic adenosine-3-monophosphate (cAMP)-dependent protein kinase is required to have any capture at all. (Middle, bottom) Local protein synthesis is necessary for the maintenance of long-term facilitation (LTF) beyond 24 h. (From Casadio et al. 1999; with permission, from Elsevier (C) 1999.) 5-HT, Serotonin; EPSP, excitatory postsynaptic potential. 


\section{K. Si and E.R. Kandel}

only from the synapse back to the nucleus (Martin et al. 1997a; Lee et al. 2007), but also from the nucleus to specific synapses.

\section{The "Synaptic Capture" Hypothesis}

How does CREB-dependent transcription in the cell body, which is accessible to all synapses, confer synapse-specific LTF? To explain how this specificity can be achieved in a biologically economical way, despite the massive number of synapses made by a single neuron, Martin et al. (1997a) and Frey and Morris (1997) proposed the "synaptic capture" hypothesis. This hypothesis, also referred as "synaptic tagging," proposed two possibilities that are not mutually exclusive. First, the products of gene expression are delivered to all of the synapses throughout the cell, but are functionally incorporated in only those synapses that have been molecularly altered or "tagged" by previous synaptic activity. Second, the products of activated transcription and translation are selectively delivered to the synapse that has been "tagged" by synaptic activity. The model of a "synaptic tag," whereby gene products are delivered to all synapses but are only used at synapses that have been tagged, has been supported by a number of studies both in Aplysia (Figs. 3 and 4) (Martin et al. 1997b; Casadio et al. 1999) and in the rodent hippo-

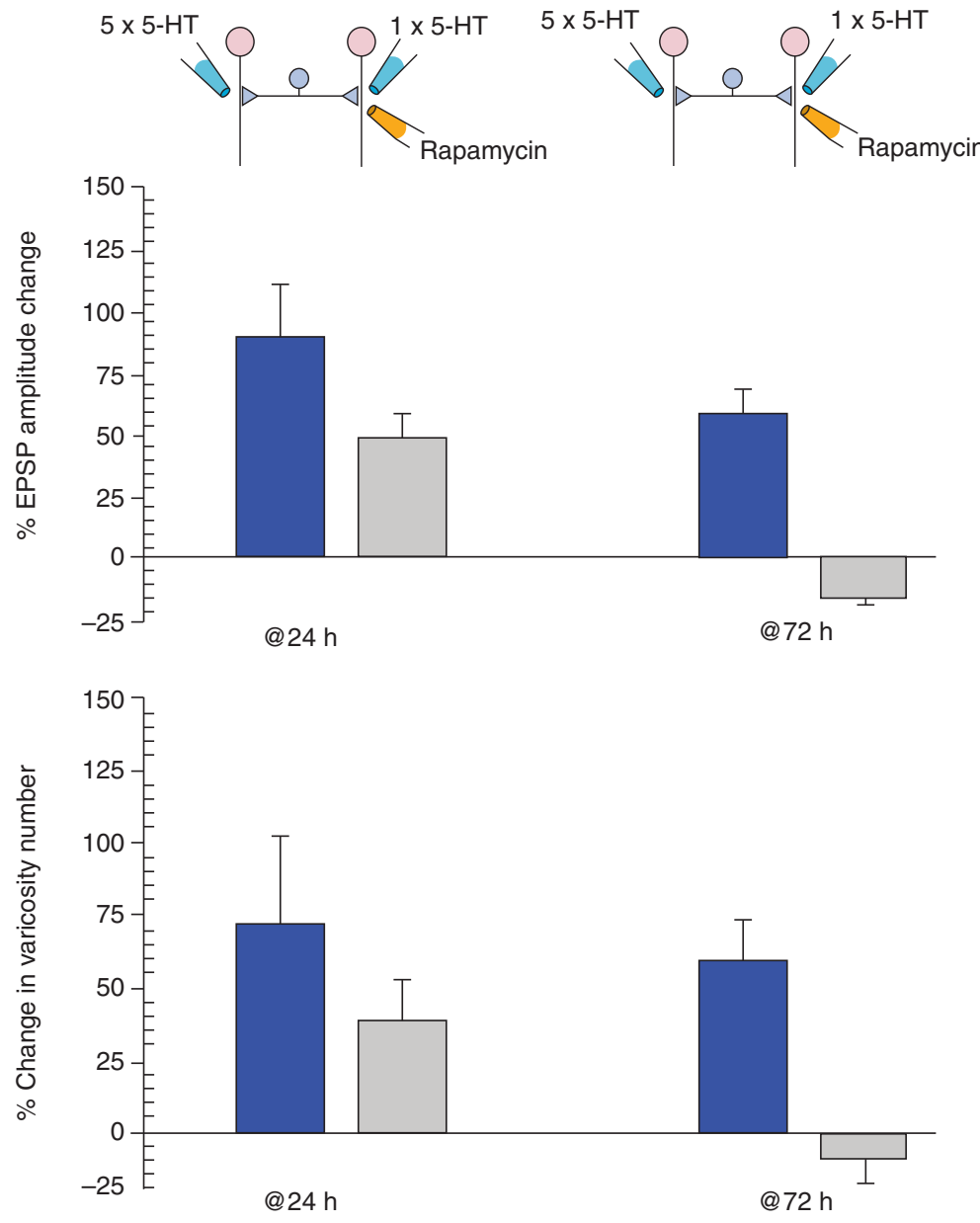

Figure 4. Rapamycin blocks the maintenance of growth at 72 h. (From Casadio et al. 1999; with permission, from Elsevier (C) 1999.) 5-HT, Serotonin; EPSP, excitatory postsynaptic potential. 
campus (Frey and Morris 1997, 1998; Barco et al. 2002; Dudek and Fields 2002).

\section{MOLECULAR MECHANISMS OF SYNAPTIC CAPTURE}

This raised the question: How is a synapse tagged? The clues as to how synapses are "tagged" came from the observations that the synapse-specific LTF requires more than the activation of CREB-driven gene transcription in the nucleus (Casadio et al. 1999). Injection of phosphorylated CREB-1 into the cell body of the sensory neuron gives rise to LTF at all the synapses. This facilitation, however, is not maintained beyond $24-48 \mathrm{~h}$, nor is it accompanied by synaptic growth. Maintenance and growth is achieved only if the synapse is also simultaneously activated by a single pulse of 5-HT.

By applying various chemical inhibitors to the synapse, Martin et al. (1997a) found two distinct components of tagging in Aplysia. The first component initiates long-term synaptic plasticity and growth, and requires the CAMPdependent protein kinase A (PKA). The second component stabilizes and maintains long-term functional and structural changes at the synapse and requires, in addition to protein synthesis in the cell body, local protein synthesis at the synapse (Figs. 4 and 5) (Martin et al. 1997a,b).

\section{The Control of Local Protein Synthesis at the Marked Synapse}

That local protein synthesis is required specifically for the persistent phase of functional and structural change of the synapse, suggested that some critical proteins are either limiting or absent in an unstimulated synapse and are made locally at the tagged synapse. Because messenger RNAs (mRNAs) are made in the cell body, and then shipped to all synapses, it suggested to Si et al. (2003a) that these mRNAs are likely to be dormant before they reach the tagged synapse. If that were true, the tag could be a regulator of translation that is capable of activating dormant mRNAs.

A search for regulators of protein synthesis in neurons that can confer both spatial and temporal restrictions led to the identification of CPEB protein (Si et al. 2003a). CPEB is a family of RNA-binding protein first discovered by Hake and Richter in Xenopus oocytes (Hake and Richter 1994). Richter and colleagues have found that oocyte maternal RNA is silent until translationally activated by CPEB (Fig. 5) and

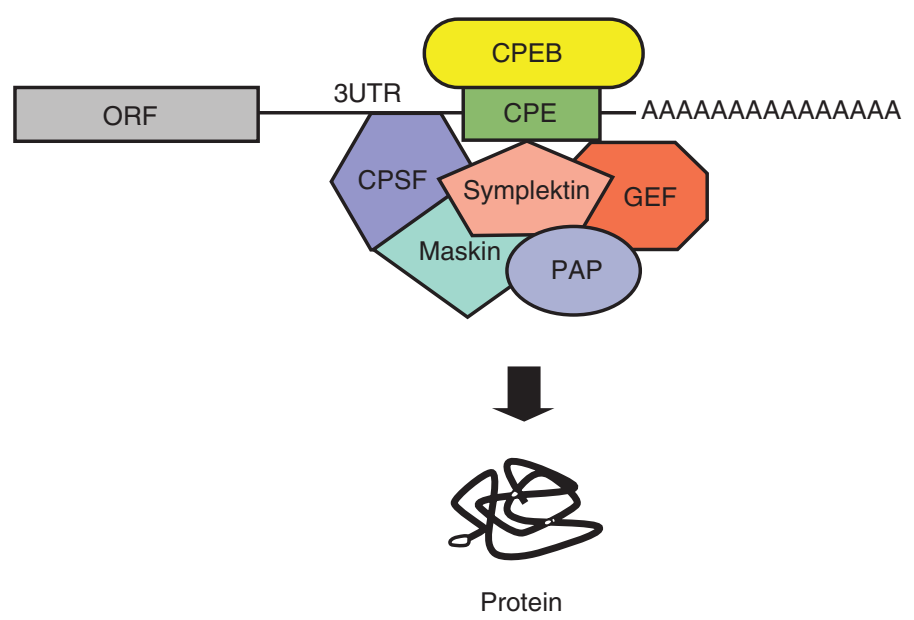

Figure 5. The cytoplasmic polyadenylation element binding protein (CPEB) binds to the cytoplasmic polyadenylation element (CPE) and activates dormant messenger RNA (mRNA). ORF, Open reading frame; UTR, untranslated region; CPSF, cleavage/polyadenylation specificity factor; GEF, guanine nucleotide exchange factor; PAP, poly(A) polymerase. 
K. Si and E.R. Kandel

CPEB activity is modulated by external signals, such as hormone progesterone.

In a search for CPEB in Aplysia, Si et al. (2003a) found that Aplysia neurons had, in addition to the developmental isoform described by Richter, a new isoform of CPEB (ApCPEB) with distinct properties. ApCPEB had three features that made it an attractive candidate for a synapse-specific tag for stabilization: (1) in response to 5 -HT, the stimulus that produces the tag, the amount of ApCPEB increases only in the stimulated synapse (Fig. 6); (2) ApCPEB regulates translation of the target mRNA and some of these mRNA targets are involved in cellular growth; and, finally, (3) blocking of ApCPEB at a tagged (active) synapse prevented the maintenance without affecting the initiation of LTF (Si et al. 2003a,b). Intriguingly, synapses in which LTF has already been induced, blocking ApCPEB blocks the persistence of that facilitated state (Miniaci et al. 2008). Thus, the maintenance of LTF over a period of days is a dynamic process requiring continuous presence of CPEB and protein synthesis (Si et al. 2003a).

\section{A Prion-Like Mechanism of CPEB Regulates Local Protein Synthesis}

Because proteins have a relatively short half-life compared with the duration of memory, structural changes at the synaptic level have been postulated to confer stability to the memory, and it was implicitly assumed that the requirement for activity-dependent molecular changes was transient. However, studies by Martin et al. and others have suggested that the maintenance of learning-related structural alterations requires ongoing macromolecular synthesis (Martin et al. 1997a; Kandel 2001). This posed a problem because there is constant turnover of proteins at the synapse. A potential solution to this problem of molecular turnover is either to have proteins with an unusually long half-life or proteins that participate in a self-sustaining biochemical reaction. In 1984, Crick first addressed the possibility of a self-sustained molecular alteration as the basis of long-term memory storage using protein phosphorylation as a candidate mechanism (Crick 1984).

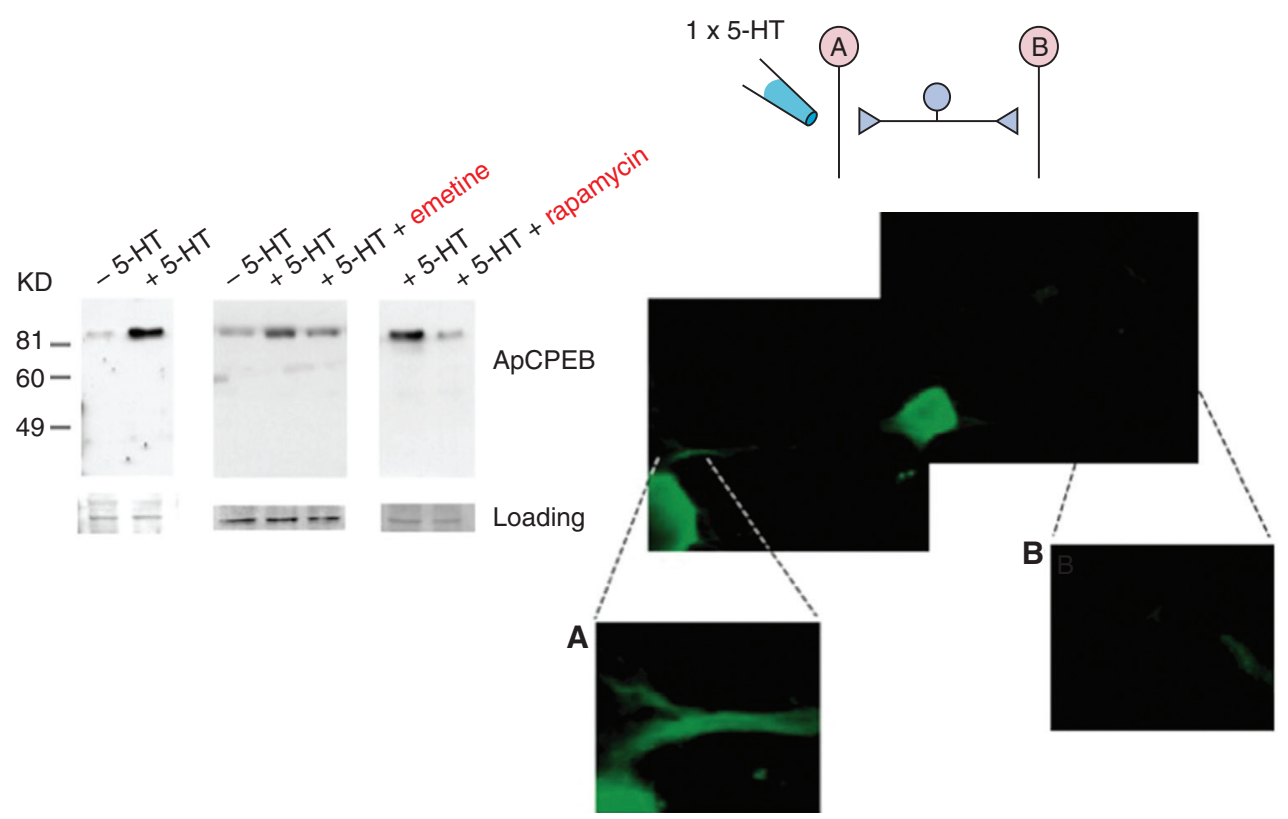

Figure 6. Aplysia cytoplasmic polyadenylation element-binding protein (ApCPEB) is induced by $1 \times 5$-HT (serotonin) in the process of the sensory neuron. (From Si et al. 2003; reprinted, with permission, from the author). KD, Knockdown. 
One plausible candidate for a self-sustaining biochemical reaction came from analysis of the amino acid sequence of ApCPEBs. In examining the sequence, $\mathrm{Si}$ et al. made the surprising observation that the neuronal isoform of ApCPEB has a glutamine and asparagine (Q- and N)-rich amino-terminal domain reminiscent of well-characterized prions in yeast (Fig. 7). Almost $50 \%$ of the first 150 amino acids were glutamine or asparagine. A search of the protein sequence database revealed putative homologs of the Aplysia neuronal CPEB in Drosophila, mouse, and human, with amino-terminal extensions of similar character.

An array of assays developed for studying prions in yeast revealed that ApCPEB could exist in yeast in two distinct conformational states, very much like other yeast PrPs. One of these states is monomeric and incapable of self-perpetuation. The other state is aggregated and stably inherited across generations in yeast, although occasional switches to the monomeric state occur (Si et al. 2003b; Heinrich and Lindquist 2011).

Si et al. (2010) went on to explore the conformational state of ApCPEB in Aplysia neu- rons. They found that when ApCPEB was overexpressed in Aplysia sensory neurons, it formed punctate structures that were amyloid-like in nature, a common characteristic of all known prions and prion-like proteins. These punctate structures were caused by self-assembly of the ApCPEB protein and once formed they could recruit newly synthesized protein, a feature necessary for self-sustenance. Intriguingly, application of five pulses of 5-HT, which produces LTF, increased the number of puncta suggesting that the aggregation of ApCPEB could be regulated by modulators of synaptic activity. Importantly, injection of an antibody that selectively binds the aggregated form of ApCPEB did not prevent the initiation of LTF but selectively blocked its maintenance beyond $24 \mathrm{~h}$ (Fig. 7).

microRNA-22 Gates Long-Term Heterosynaptic Plasticity in Aplysia through Presynaptic Regulation of CPEB and Downstream Targets

It soon became apparent that the aggregation of ApCPEB is critical for maintenance and can be regulated by $5-\mathrm{HT}$. This raised the ques-

A
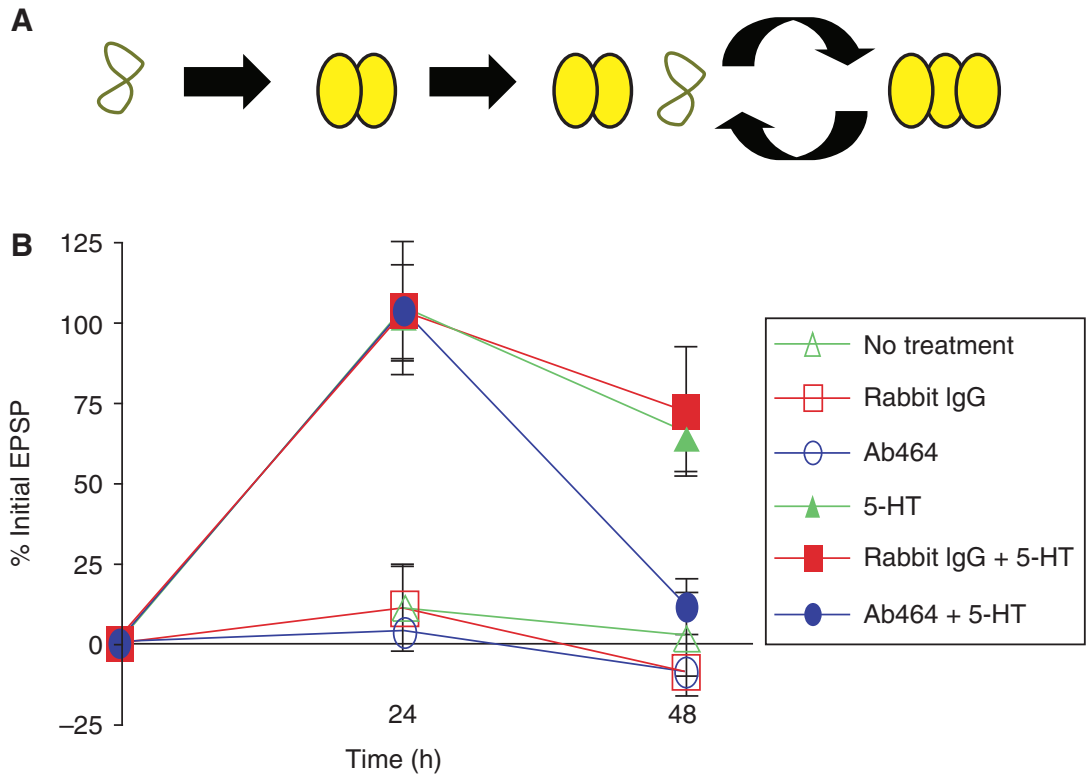

Figure 7. Injection of antibodies that specifically recognize the aggregated form of Aplysia cytoplasmic polyadenylation element-binding protein (ApCPEB) (Ab464) blocks persistence of long-term facilitation (LTF). 5-HT, Serotonin; EPSP, excitatory postsynaptic potential (From Si et al. 2010; reprinted, with permission, from the author.) 
tion: How does the heterosynaptic modulator 5-HT-which induces learning-related longterm plasticity of sensorimotor neuron synapses-determine the initial up-regulation, and the consequent conversion of CPEB into its aggregated state in the presynaptic sensory neurons? In search of a mechanism whereby 5-HT up-regulates the levels of ApCPEB in sensory neurons, Fiumara et al. (2015) identified an Aplysia microRNA 22 (miR-22) that is expressed in sensory neurons. MiR-22 is down-regulated by 5-HT and inhibition of miR-22 increases CPEB levels and enhances LTF, mimicking the effect of 5-HT-dependent up-regulation of CPEB. Conversely, the presynaptic overexpression of miR-22 impairs LTF, mimicking the physiological effect of CPEB down-regulation.

Fiumara et al. next asked, how does miR-22 effect ApCPEB aggregation? They found, interestingly, that miR-22 overexpression also indirectly down-regulates presynaptic expression of Aplysia protein kinase C (aPKC), most likely via its effect on CPEB, because aPKC 3'UTR (untranslated region) contains several cytoplasmic polyadenylation elements (CPEs). Earlier studies in Drosophila also identified the Drosophila aPKC mRNA as a target for Orb2, the ortholog of Aplysia CPEB (Mastushita-Sakai et al. 2010). The aPKC/M has an established function in synaptic plasticity in the postsynaptic compartment, both in vertebrates and in Aplysia (Sacktor 2011; Bougie et al. 2012). Using genetic approaches based on the selective presynaptic overexpression of wild-type and dominant negative forms of the kinase, Fiumara et al. found that aPKC also acts presynaptically in the maintenance of LTF. Taken together, these results support a model in which the presynaptic 5-HT-dependent down-regulation of miR-22 promotes the up-regulation and activation of $\mathrm{CPEB}$ and downstream targets, including an atypical PKC. As discussed below in the context of Drosophila Orb2, phosphorylation may modulate aggregation of CPEB. Therefore, the activation of aPKC may act synergistically with CPEB or directly affect CPEB to maintain learning-related long-term synaptic facilitation.
An activity-dependent prion-like switch controlled by 5 -HT acting through miR-22 could, therefore, serve as a mechanism to maintain a self-sustained activated molecular state. According to this model (Fig. 11), ApCPEB in the sensory neuron has at least two conformational states: (1) a recessive monomeric state, in which ApCPEB is inactive or acts as a repressor of translation; and (2) a dominant, selfsustaining, active multimeric state. In a naïve synapse, the basal level of ApCPEB is low and the protein is in the monomeric state. An increase in the amount of ApCPEB induced by inhibition of miR-22 by $5-\mathrm{HT}$, results in the conversion of ApCPEB from the monomeric to an aggregated prion-like state, which might be more active or be devoid of the inhibitory function of the basal state. Once the prion state is established at an activated synapse, dormant mRNAs, made in the cell body and distributed globally to all synapses, can be activated only locally, through the local activation of ApCPEB. Because the activated ApCPEB can be self-perpetuating, it can contribute to a self-sustaining synapse-specific long-term molecular change and provide a mechanism for the stabilization of learning-related synaptic modification and growth, and contribute to the persistence of memory storage (Fig. 8).

In addition to regulating the level of expression, are there other means for regulating the autocatalytic, self-perpetuating chemical reaction resulting in synaptic enhancement? One possibility is that molecular chaperones might regulate the conformational states of ApCPEB. Indeed, Si et al. (2003b) found that the heat shock protein HSP104 is capable of reversing the active form of ApCPEB in yeast. A second mechanism of regulation is through the restriction of the action of ApCPEB to specific synapses. If ApCPEB in the active state could escape from a newly potentiated synapse, the entire cellular ApCPEB would then be converted to an active state, thereby leading to erroneous potentiation of all synapses. Thus, there must be a mechanism that effectively restricts the activated ApCPEB at the potentiated synapse. One possibility is that the polymerization of ApCPEB creates a diffusion barrier restricting 


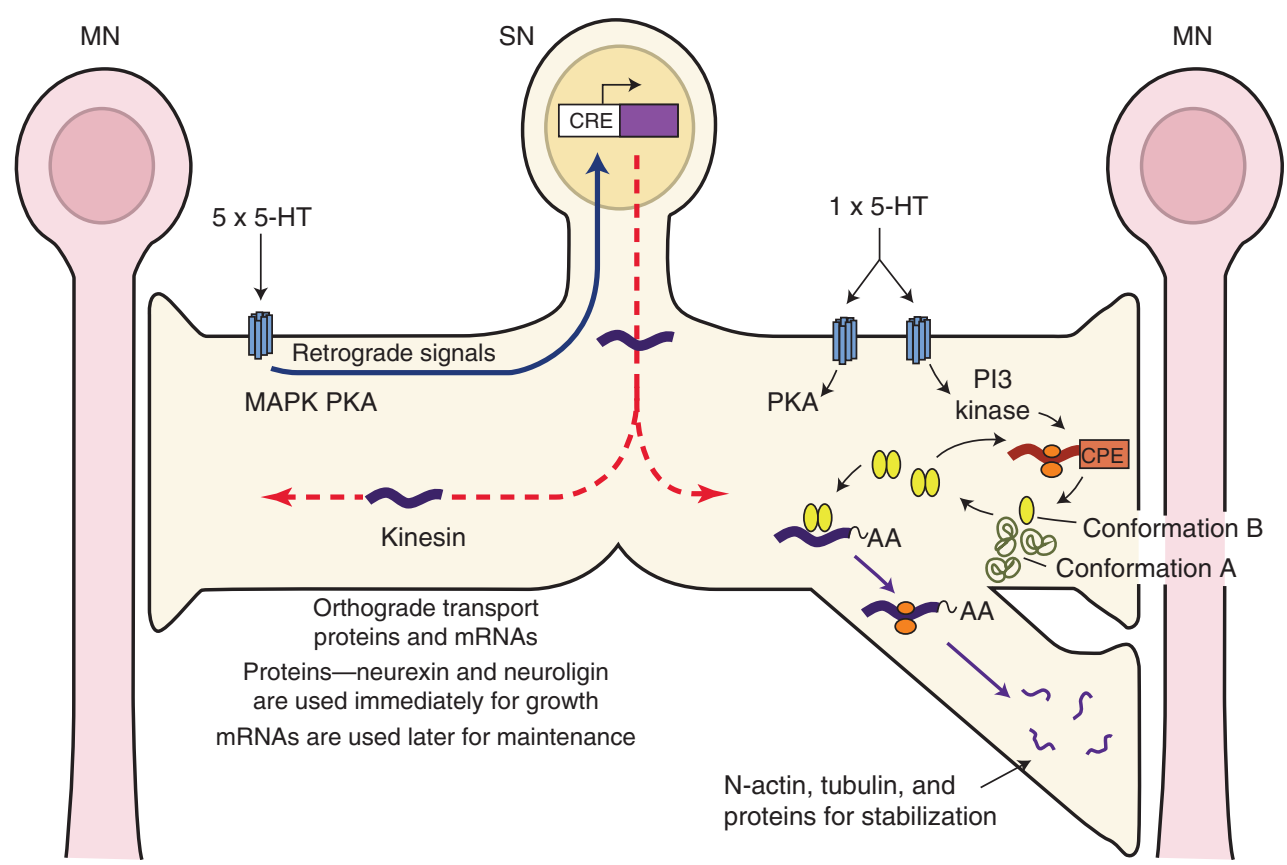

Figure 8. A model for the initiation and persistence of long-term memory storage. A single pulse of serotonin $(5-\mathrm{HT})$ to sensory neuron $(\mathrm{SN})$ and motor neuron $(\mathrm{MN})$ synapses recruits the short-term process, and this requires protein kinase A (PKA) activity. PI3 kinase also becomes activated and stimulates cytoplasmic polyadenylation element binding protein (CPEB)-dependent translation. Five pulses of 5-HT activate cyclic adenosine-3-monophosphate (cAMP)-response element-binding protein 1 (CREB-1) in the nucleus through the PKA-mitogen-activated protein kinase (PKA-MAPK) pathway and facilitate enhanced, kinesin-mediated fast axonal transport of proteins, mRNAs, and organelles to synapses. These activation steps are critical for the initiation of long-term facilitation (LTF). RNAs transported by kinesin may be used for persistence. During the persistence phase, CPEB at the stimulated synapse activates polyadenylation of cytoplasmic polyadenylation element (CPE)-containing RNAs through a prion-like mechanism for the self-perpetuation of synapse-specific memory storage. (From Puthanveettil and Kandel 2011; reprinted, with permission, from the authors.)

the protein at the activated synapse. Compartmentalization of activated ApCPEB could also be facilitated through interactions of its polymers with other cellular components, including those that are translated on ApCPEB activation. For example, the actin cytoskeleton is involved in the formation, propagation, and intracellular localization of the yeast prion $\left[\mathrm{PSI}^{+}\right]$(Ganusova et al. 2006), and oligomeric ApCPEB has been found to activate translation of actin mRNAs (Si et al. 2003a).

\section{ApCPEB Homolog Also Exists in Drosophila}

The proposed critical role and mode of action of CPEB in the maintenance of long-term plasticity and hence memory is not restricted to Aply- sia. The Drosophila CPEB family is comprised of two genes: Orb1 and Orb2. Orb1 is an ortholog of mammalian CPEB1 and Orb2 belongs to the mammalian CPEB2-3 family (Keleman et al. 2007). All family members share common RNA binding motifs in the carboxy-terminal end but differ significantly in the amino-terminal end, suggesting functional differences among isoforms. Similar to the ApCPEB, Drosophila $\mathrm{CPEB}$, Orb2, carries a prion-like domain at the amino-terminal end and the Orb2 prion domain can functionally substitute the canonical prion domains of yeast prion Sup35 (R Hervás, L Li, and M Carrión-Vázquez, unpubl.).

Similar to ApCPEB, Orb2 has two distinct physical states in the adult fly brain: a mono- 
meric state and a stable sodium dodecyl sulfate (SDS)-resistant amyloid-like oligomeric state. The oligomers are formed at physiological concentrations of Orb2 protein and stimulation of behaviorally relevant neurons, such as the Kenyon cells of the mushroom body or octopamine or dopamine-responsive neurons, increase the level of the oligomeric Orb2 (Krüttner et al. 2012; Majumdar et al. 2012). Considering the evolutionary distance between the Aplysia CPEB and Drosophila Orb2, these observations suggested that the amyloidogenic oligomers of Orb2/CPEB may act to stabilize activity-dependent changes in synaptic efficacy across species.

Role of Prion-Like State of Drosophila Orb2 in the Persistence of Memory

Is the prion-like conversion of Drosophila Orb2 important for long-lasting memory? Keleman et al. (2007) found that when the Q-rich 80 amino acids from the prion-like domain of Drosophila Orb2 were removed, the flies had no problem in forming short-term male courtship-suppression memory; however, the flies failed to form any long-term memory. These observations suggested that the prionlike domain is important for long-term memory. To test whether the prion-like conversion is necessary for long-term memory, Majumdar et al. (2012) created a specific mutant variant of Orb2. The Drosophila Orb2 has two protein isoforms: Orb2A and Orb2B. Both isoforms contain an RNA-binding domain, a zinc finger domain, and an amino-terminal prion-like domain, but amino acid sequences upstream of Qrich sequence are different. The short Orb2A isoform has eight and the longer isoform Orb2B has 162 amino acids upstream of the prion-like domain. These two isoforms also differ in their biophysical properties. Orb2A forms amyloids more efficiently than Orb2B both in vitro and in vivo. Although present at a very low level, deletion of Orb2A prevented Orb2B oligomerization and in the adult brain Orb2A and Orb2B forms hetero-oligomers. These observations suggested that the rare Orb2A protein either acts directly as a seed to induce activitydependent oligomerization of the abundant
Orb2B protein or Orb2A oligomerization indirectly affects oligomerization of Orb2B. The seeded oligomerization of Orb2 was further supported by Krüttner et al. (2012), who observed that the prion-like domain of Orb2A is sufficient for long-term memory as long as there is full-length Orb2B protein. Majumdar et al. (2012) performed a random mutagenesis screen and identified a number of mutations in the eight amino acids that are unique to the Orb2A isoform that prevented Orb2A oligomerization. One of these point mutations in Orb2A, Orb2AF5 $>$ Y5, reduced activity-dependent amyloid-like oligomerization of Orb2 when introduced into the fly. Notably, flies carrying this mutation showed a very specific memory deficit (Fig. 9). In two different behavioral paradigms, male courtship-suppression memory and appetitive associative memory, the memory score of mutant flies were similar to the memory score of wild type a day after training. However, unlike wild type flies, the mutants memory begins to decay at $2 \mathrm{~d}$ and by $3 \mathrm{~d}$ there was no measureable memory. The loss of memory in the Orb2 mutant that cannot form Orb2 amyloid is reminiscent of the loss of synaptic facilitation in sensorimotor neuron synapse on inhibition of the ApCPEB oligomers via antibody.

The seeded prion-like conversion also suggested that the amount and localization of Orb2A protein is likely to be a key determinant of when and where amyloid-like conversion would occur. Therefore, a key question in the regulation of Orb2 prion-like conversion becomes: how is the expression of the of Orb2A protein regulated? In an effort to understand the regulation of Orb2 prion-like conversion, White-Grindley et al. (2014) noticed that Orb2A protein has a very short half-life $(\sim 1 \mathrm{~h})$ consistent with its low abundance. Transducer of Erb2 or Tob, a previously known regulator of SMAD (small body size and mothers against decapentaplegic)-dependent transcription (Yoshida et al. 2000, 2003) and CPEB-mediated translation (Hosoda et al. 2011) associates with both Orb2A and Orb2B, but increases the half-life of only Orb2A. Both Orb2 and Tob are phosphoproteins and PP2A controls the phosphorylation status of Orb2A and Orb2B. Stim- 
Prion-Like Proteins and Memory

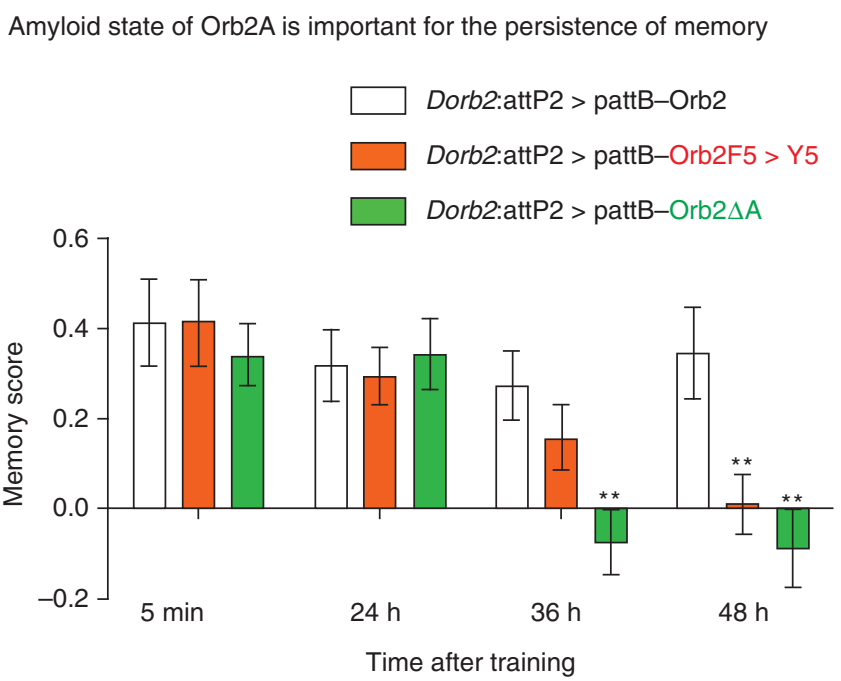

Figure 9. Orb2A is required for stable memory formation. The Orb2A mutations were introduced into a genomic fragment, and the mutant flies were generated by introducing the genomic fragments into an orb2 null background. Deletion of Orb2A or a mutation that prevents Orb2A oligomerization (F5 > Y5) selectively interferes with persistence of male courtship-suppression memory beyond a day.

ulation with tyramine or activation of mushroom body neurons enhances the association of Tob with Orb2A and Tob promotes Orb2 phosphorylation by recruiting Lim kinase (LimK). Phosphorylation destabilizes Orb2 associated Tob, whereas it increases the monomeric Orb2A protein level.

Protein phosphatase 2A (PP2A), an autocatalytic phosphatase, is known to act as a bidirectional switch in activity-dependent changes in synaptic activity (Mulkey et al. 1993; Kikuchi et al. 2003; Belmeguenai and Hansel 2005; Pi and Lisman 2008). PP2A activity is down-regulated on induction of long-term potentiation (LTP) of hippocampal CA1 synapses and up-regulated during long-term depression (LTD) (Pi and Lisman 2008). Similarly, LimK, which is synthesized locally at the synapse (Schratt et al. 2006) in response to synaptic activation, is also critical for long-term changes in synaptic activity and synaptic growth (Meng et al. 2002).

These observations suggested a model, in which in the basal state synaptic PP2A keeps the available Orb2A in a un- or hypophosphorylated and thereby unstable state. On stimulation the Tob protein that is constitutive- ly present at the synapse binds to and stabilizes the un- or hypophosphorylated Orb2A and recruits the activated LimK to the Tob-Orb2 complex, allowing Orb2 phosphorylation. Concomitant decreases in PP2A activity and phosphorylation by other kinases increases Orb2A half-life. The increase in Orb2A level as well as change in phosphorylation may induce conformational changes in Orb2A, which allows Orb2A to act as a seed. Alternatively, accumulation and oligomerization of Orb2A creates an environment that is conducive to overall Orb2 oligomerization. Phosphorylation of Orb2 may allow integration of various signaling pathways allowing Orb2 prion-like conversion to act as an activity-dependent molecular "switch."

\section{EXPLICIT MEMORY STORAGE IN MAMMALS AND THE CYTOPLASMIC POLYADENYLATION ELEMENT BINDING PROTEIN 3}

\section{CPEB3 Is a Prion-Like Protein}

Is a prion-like state of $\mathrm{CPEB}$, which is important for LTF in Aplysia and long-term memory in Drosophila, also important for synaptic plasticity and various forms of memory in mammals? 
In mouse and in human, there are four distinct CPEB genes: CPEB1 to CPEB4 (Theis et al. 2003). Although all CPEB isoforms in mammals share a similar RNA-binding domain, they differ significantly in the amino-terminal domain. Among these isoforms CPEB1, is the most well characterized and is involved in some form of LTP (Alarcon et al. 2004). Among the other CPEB genes, CPEB2 and CPEB3 have Q/Nrich regions reminiscent of the $A \mathrm{PCPEB}$ and Orb2 amino-terminal domain (Fig. 10) (Theis et al. 2003). More recent studies (Chen and Huang 2012) have revealed that two small aggregation-prone regions are also present in CPEB2 (Fig. 10), but the protein has not yet been studied from a functional point of view. Only the contribution of the amino-terminal domain of CPEB3 has so far been functionally interrogated at the molecular and behavioral level.

To explore the potential prion-like property of CPEB3, Stephan et al. (2015) expressed CPEB3 in yeast and found that it displayed the two essential features of a prion-like protein: (1) it forms amyloidogenic aggregate, and (2) aggregates are heritable across cell division. Interestingly, consistent with these findings, Krüttner et al. (2012) recently found that the CPEB3 Q-rich amino terminus can functionally substi- tute the Orb2A prion-like domain in Drosophila. Like ApCPEB, Orb2A has been found to behave like a prion in yeast ( $\mathrm{Si}$ et al. 2003a) and to form amyloid-like aggregates that mediate the persistence of memory (Krüttner et al. 2012; Majumdar et al. 2012). Moreover, deletion analysis of the prion domain of CPEB3 revealed a novel, tripartite organization consisting of two Q/N-rich aggregation-promoting domains surrounding a regulatory module that mediates an interaction between CPEB3 and the actin cytoskeleton. Although the functional significance of the interaction between CPEB3 and actin is ongoing, nonetheless these data provide evidence that CPEB3 has the capacity to undergo a prion-like conversion.

\section{THE ROLE FOR CPEB3 AGGREGATES IN LOCAL PROTEIN SYNTHESIS}

Does a prion-like conversion of CPEB3 evident in yeast also occur in the brain and, if so, what function does it serve? Fioriti et al. (2015) found that in the basal state CPEB3 binds to and represses the translation of its target mRNAs in the brain, such as the $\alpha$-amino-3-hydroxy-5methyl-4-isoxazolepropionic acid (AMPA) receptor subunits GluA1 and GluA2 (Huang

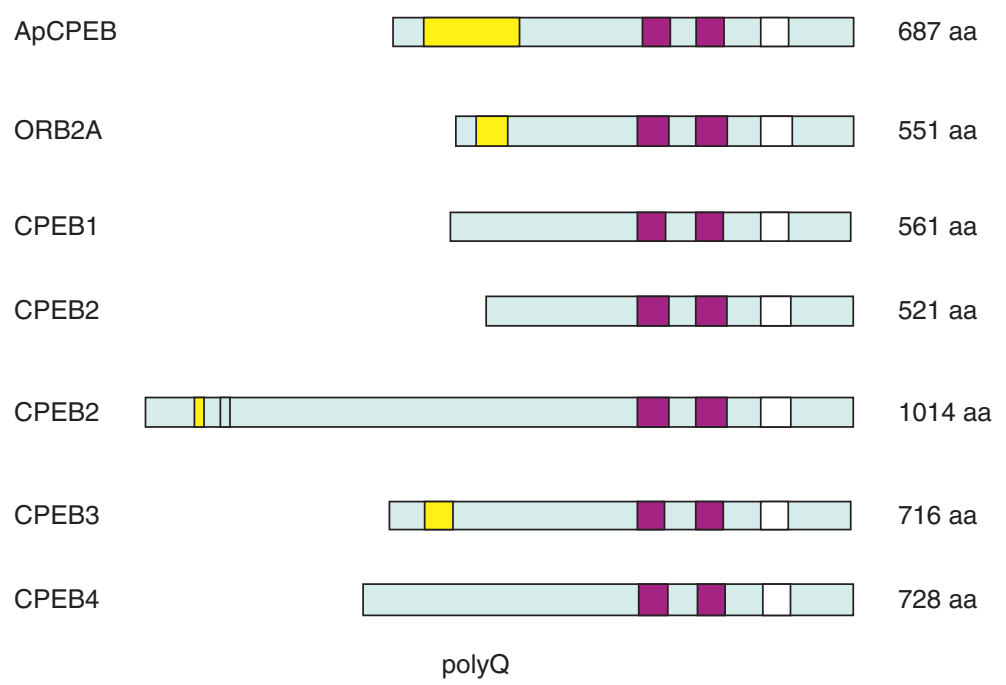

Figure 10. Mice have four isoforms of cytoplasmic polyadenylation element-binding protein (CPEB): CPEB1 to CPEB4. The amino-terminal domain of CPEB3 most resembles the prion-like domain of Aplysia CPEB (ApCPEB). aa, Amino acid. 
A

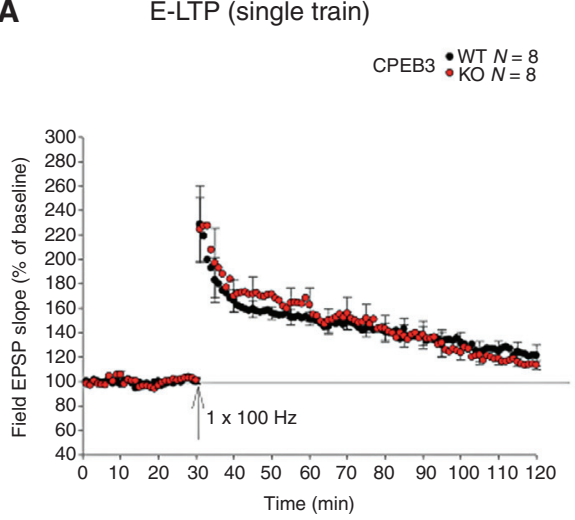

B

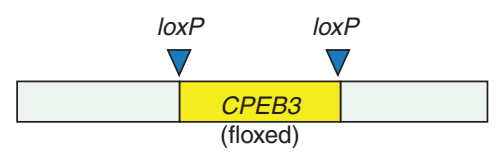

L-LTP (four train)
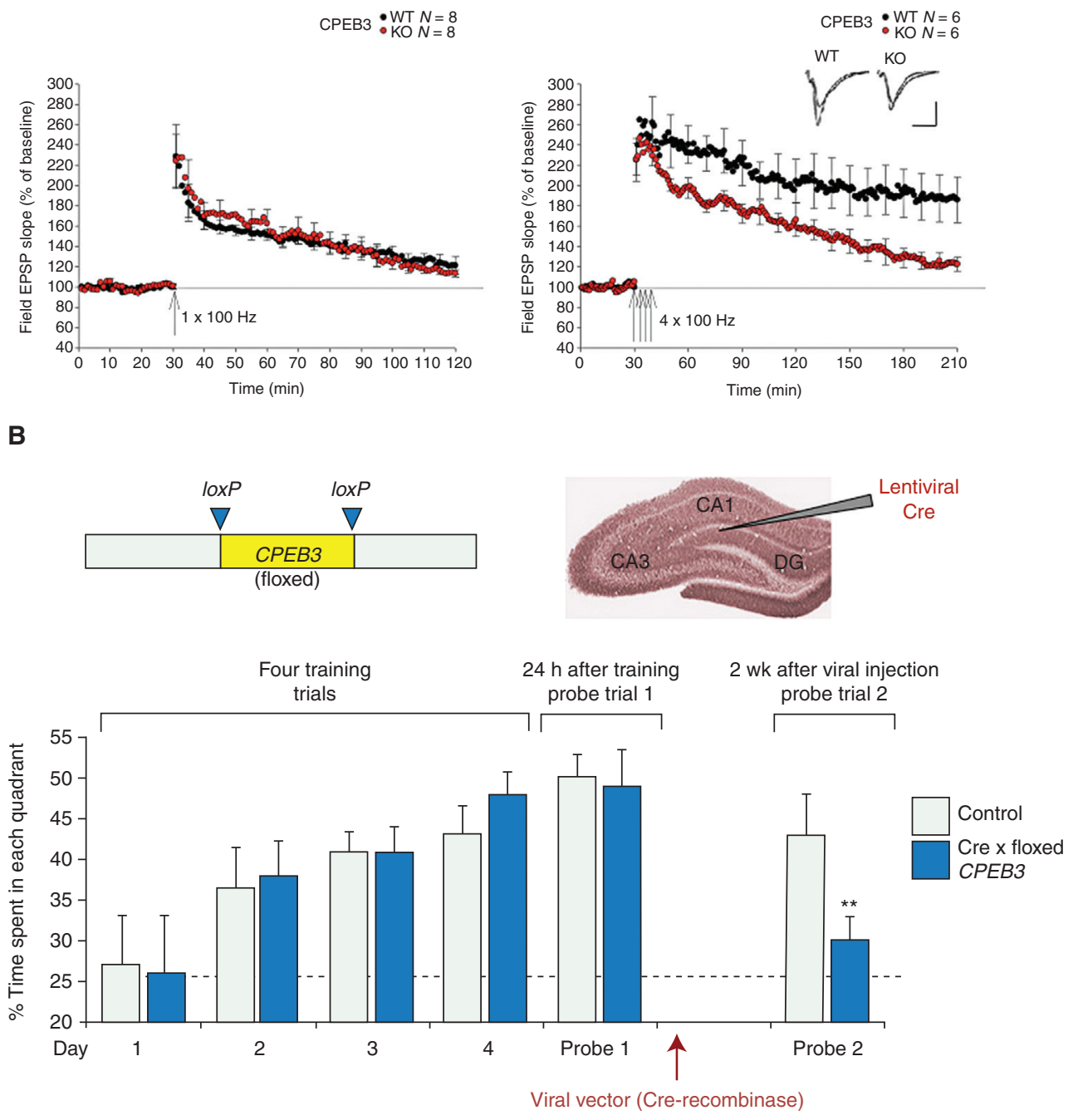

Figure 11. cytoplasmic polyadenylation element-binding protein 3 (CPEB3) conditional knockout mice show impaired $(A)$ maintenance of dopamine-dependent synaptic plasticity, and $(B)$ long-term memory. LTP, longterm potentiation; WT, wild type; KO, knockout; DG, dentate gyrus.

et al. 2006; Pavlopoulos et al. 2011). In turn, CPEB3 promotes the translation of the AMPA receptor (AMPAR) following monoubiquitination by the ubiquitin ligase neuralized (Pavlopoulos et al. 2011). Together, these data suggest that CPEB3 can act as a repressor in the basal state and can be converted to an activator in aggregation by posttranslational modification.

This raised the question: How does CPEB3 switch from one state to the other from being a repressor to being an activator of translation?
Similarly, to what has been observed in Aplysia and Drosophila, Fioriti et al. found that mouse CPEB3 form aggregates on synaptic activation in culture as well as performing a behavioral task in vivo. Moreover the dual role in translation, the switch from repression to activation, is correlated with change of CPEB3 from a soluble to an aggregated form. The propensity of CPEB3 to form aggregates derive from its amino terminus domain, which comprises, as we have seen, two regions rich in glutamine and a low 
complexity sequence, which is predicted to be poorly structured and to form aggregates (Fiumara et al. 2010).

The Persistence of Synaptic Plasticity and Memory Storage Requires CPEB3-Mediated Protein Synthesis in the Hippocampus

To determine the role of CPEB3 in the persistence of synaptic plasticity and memory, Fioriti et al. (2015) generated a conditional knockout strain of CPEB3 and surveyed the contribution of CPEB3 to the maintenance of memory. They found that CPEB3-mediated protein synthesis is required for maintenance, but not for memory acquisition (Fig. 11). The memory deficit is observed in two different behavioral paradigms, spatial object recognition and the Morris water maze task, suggesting that CPEB3-mediated processes are required for different types of hippocampal-based spatial learning tasks. Fioriti et al. (2015) also found that CPEB3 loses its ability to maintain long-term synaptic plasticity and long-term memory if its prion-like amino terminus domain is deleted. Fioriti et al., therefore, proposes that, like the Aplysia CPEB and the Drosophila Orb2A, CPEB3 can sustain the persistence of memory through a stimulus-induced conformation change, which causes protein aggregation and a change in function that allows enhanced translation of CPEB3 target mRNAs, such as the AMPAR subunits GluA1 and GluA2.

These results provide the first evidence for a prion-like mechanism to sustain memory in the mouse brain during consolidation and maintenance.

SUMOylation Inactivates, whereas Ubiquitination Activates CPEB3

The dominant nature of the CPEB3 aggregates characteristic of other CPEB-related functional prions led Drisaldi and colleagues (2015) to search for inhibitory constraints that might be important in regulating aggregate formation. They found that small-ubiquitin-like modifier or SUMOylation of CPEB3 acts on an inhibitory constraint. In its basal state, CPEB3 is SUMOylated in hippocampal neurons and in its SU-
MOylated form CPEB3 is monomeric and acts as a repressor of translation. Following neuronal stimulation, CPEB3 is converted into an active form, which is associated with a decrease in SUMOylation and an increase of aggregation. A chimeric CPEB3 protein fused to SUMO prevents the protein from aggregating and from activating the translation of target mRNAs. These findings suggest a model whereby SUMO regulates translation of $\mathrm{mRNAs}$ and synaptic plasticity by modulating the aggregation of CPEB3

Because SUMOylation keeps it in an inactive state, what activates CPEB3? Pavlopoulos et al. found that CPEB3 is activated by Neuralized1, an E3 ubiquitin ligase (Pavlopoulos et al. 2011). CPEB3 interacts with Neuralized1 in dendrites of adult hippocampal neurons. In mice overexpressing Neuralized1, specifically in the forebrain, the levels of monomeric CPEB3 are increased in the hippocampus, whereas CPEB1 and CPEB4 are unaffected. Pavlopoulos et al. found that CPEB3 interacts with Neuralized1 via its amino-terminal, prion-like domain, and that this interaction leads to the monoubiquitination and consequent activation of CPEB3. Strikingly, overexpression of Neuralized1 activates $\mathrm{CPEB} 3$ in cultured hippocampal neurons (Fig. 12).

These results suggest a model whereby Neuralized1-mediated ubiquination facilitates hippocampal plasticity and hippocampal-dependent memory storage by modulating the activity of CPEB3 and CPEB3-dependent protein synthesis. In response to synaptic activity, the protein levels of Neuralized 1 are increased, leading to the ubiquination and activation of CPEB3, and consequent production of synaptic components critical for the formation of new functional synaptic connections. Because CPEB3 can be SUMOylated as well as ubiquitinated the relationship between these two posttranslational modification is of interest.

Finally, although the evidence for a plausible role of prion-like conversion of neuronal $\mathrm{CPEB}$ in long-lasting memory is growing, still several important questions remain unanswered. Does persistence of memory require continued presence of the prion-like state? Does decay of memory coincide with the dis- 

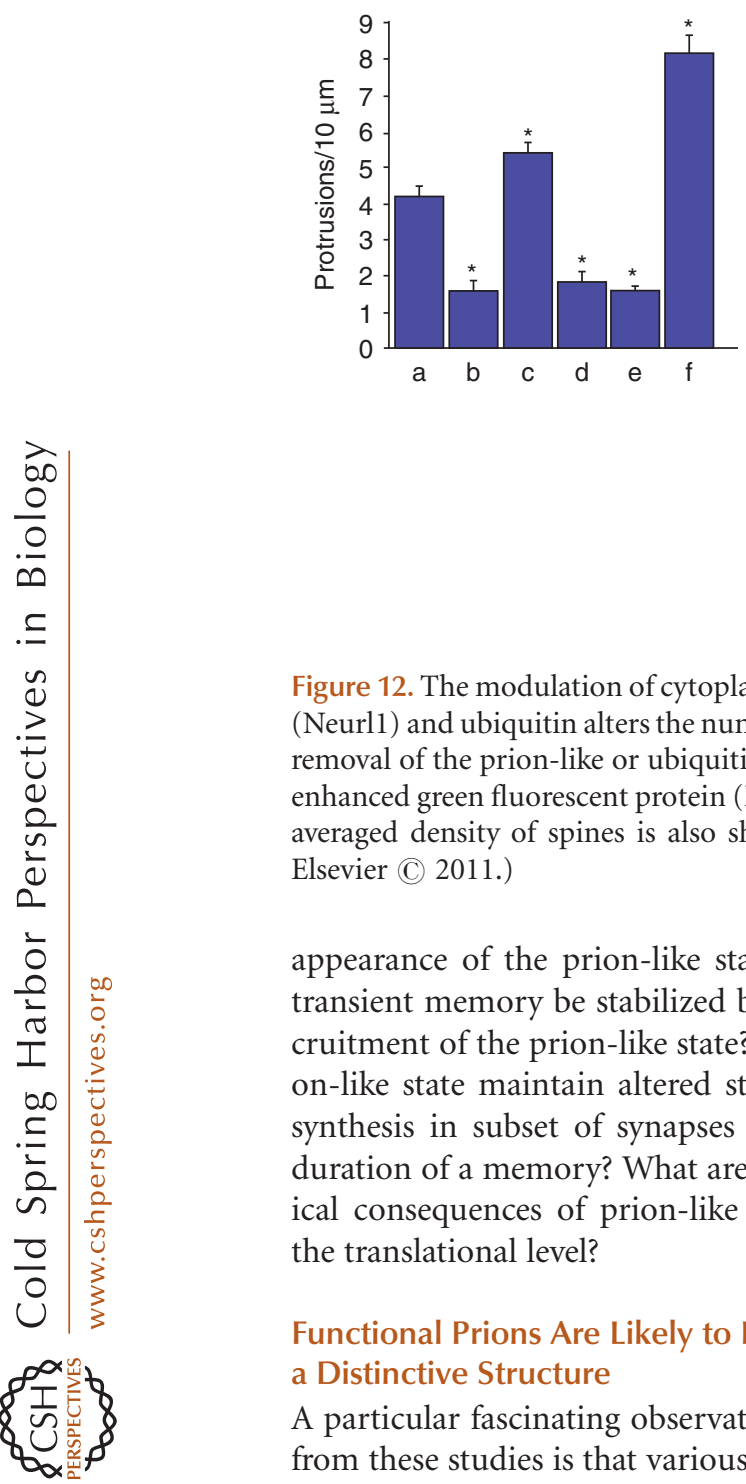
Elsevier (C) 2011.) a

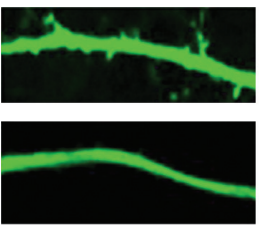

c

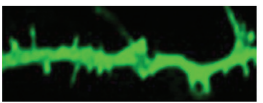

d
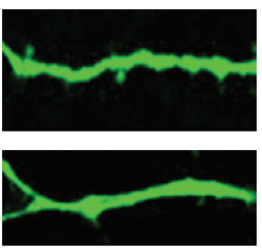

$f$

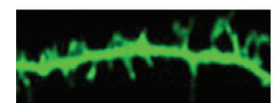

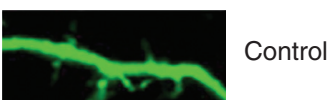
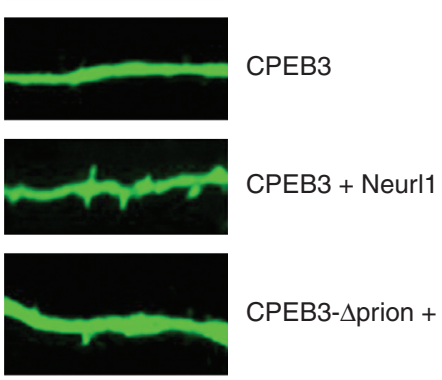

CPEB3- $\Delta$ prion + Neurl1

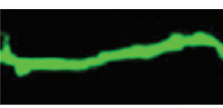

CPEB3 + Neurl1 ${ }^{\text {Rm }}$

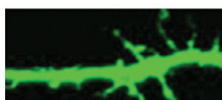

CPEB3-Ub

Figure 12. The modulation of cytoplasmic polyadenylation element-binding protein 3 (CPEB3) by Neuralized1 (Neurl1) and ubiquitin alters the number of spines in cultured hippocampal neurons. Modulation is blocked by removal of the prion-like or ubiquitin ligase domains. Dendrites of cultured hippocampal neurons expressing enhanced green fluorescent protein (EGFP) alone (control) or EGFP and the indicated proteins are shown. The averaged density of spines is also shown. (From Pavlopoulos et al. 2011; reprinted, with permission, from

appearance of the prion-like state, and can a transient memory be stabilized by artificial recruitment of the prion-like state? Does the prion-like state maintain altered state of protein synthesis in subset of synapses for the entire duration of a memory? What are the biochemical consequences of prion-like conversion at the translational level?

\section{Functional Prions Are Likely to Have a Distinctive Structure}

A particular fascinating observation to emerge from these studies is that various species CPEB in Aplysia, Drosophila, and mice can form functional prionogenic aggregates in the mature adult neurons that support memory, whereas other prions and amyloids formed by a number of other proteins in the nervous system causes cognitive deficit and neurodegeneration. How can these two diametrically opposite outcomes be reconciled? The simplest and most likely answer is some inherent structural differences between functional and toxic amyloids and prions. However, the specific structural and molecular features, if any, that distinguish functional prions from pathological prions are currently not known.

Conventional prions have been found to have a $\beta$-sheet-rich structure, and the structural transition from soluble to aggregated forms occurs through uncontrolled structural transitions and consequent misfolding and aggregation. With functional prions, such as Aplysia CPEB, Drosophila Orb2, and CPEB3, the conversion from one state to another is regulated by physiological signals. To search for a difference between the two classes of prions-pathogenic and functional-Fiumara et al. (2010) searched for other types of structures, and found that in addition to $\beta$-sheet there are ApCPEB coiledcoil $\alpha$ helices that can mediate prion-like oligomerization. However, unlike $\beta$-sheets, coiledcoils are responsive to environmental signal and, therefore, regulatable (see Fiumara et al. 2010).

Raveendra et al. (2013) next performed more in depth structural studies on ApCPEB using functional nuclear magnetic resonance (NMR). Consistent with studies of Fiumara et al., these functional NMR studies revealed that the prion-like domain is not solely com- 


\section{K. Si and E.R. Kandel}

posed of $\beta$-sheet but also has a novel mixed structure containing helical and random coil stretches. This mixed structure view is consistent with bioinformatics and mutagenesis studies (Fiumara et al. 2010) that predicted that the glutamine rich prion domain of ApCPEB has a propensity to form $\alpha$-helical coiled-coil structures. This "mixed structure model" (Fig. 13) has a plausible advantage in that it might allow the $\beta$-sheet to form a fiber axis scaffold. In turn, this would allow the carboxy-terminal domains to be stacked together yet be exposed and free to bind mRNA on the surface of the $\beta$-sheet fiber axis scaffold. This mixed structure model would allow the coordinated translation of the population of interrelated mRNAs required for the stabilization of synaptic growth.

\section{An Overall View: Functional Prions in Perspective}

The realization that protein conformational switches could provide a means for inheritance of phenotypes dates back $>20$ years (Wickner 1994). Although prions were initially discovered as infectious proteinaceous agents that are associated with a class of fatal degenerative diseases of the mammalian brain, the discovery of fungal prions-which are not associated with disease-first suggested that the effects of prion mechanisms on cellular physiology could be viewed in a different light. Fungal prions as epigenetic determinants alter a range of cellular processes, including metabolism and gene expression (Tompa and Friedrich 1998; Eaglestone et al. 1999; True and Lindquist 2000; True et al. 2004; Halfmann et al. 2012). These changes lead to a variety of prion-associated phenotypes. The data we review here provides one of the early examples of the existence of a prion-like protein conformational switch in the brain that may instead of causing loss of memory allow stabilization of memory. The mechanistic similarities between prion-like propagation of CPEBs in snails, flies, and mammals suggest that prions are not a biological anomaly, but, instead, could perhaps embody a ubiquitous regulatory mechanism. Indeed, functional prion-like protein or other self-assembling proteins have now been found in other species including human (Hou et al. 2011). Therefore, it is tempting to speculate that in the nervous system there might be other proteins in addition to CPEB that serve normal physiological functions

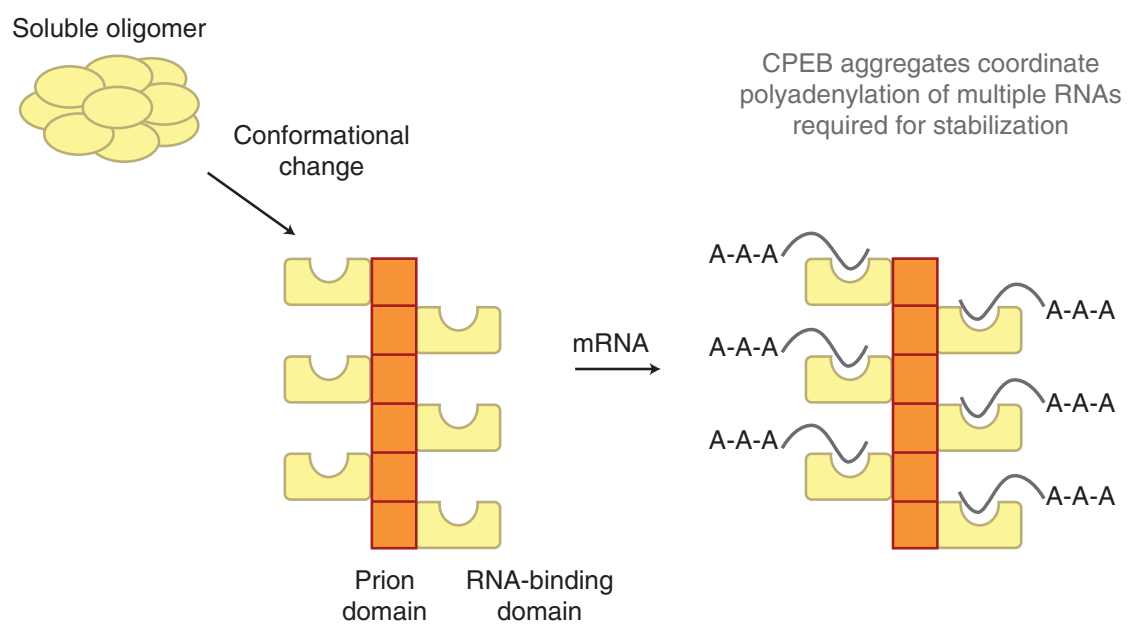

Figure 13. A mixed structure model for prion-like aggregation of Aplysia cytoplasmic polyadenylation elementbinding protein (ApCPEB) and protein synthesis. The prion domains are stacked together, leaving the RNAbinding domain exposed, and free to bind messenger RNAs (mRNAs) on the surface of the $\beta$-sheet fiber-axis scaffold. This can allow the coordinated translation of the population of interrelated mRNAs required for stabilization of synaptic growth (based on Raveendra et al. 2013). 
at the prion state and perhaps the preponderance of amyloid-based disease in the nervous system is linked to the presence of functional prions in the nervous system.

\section{REFERENCES}

Aguzzi A, Weissmann C. 1998. Prion diseases. Haemophilia 4: 619-627.

Alarcon JM, Hodgman R, Theis M, Huang YS, Kandel ER, Richter JD. 2004. Selective modulation of some forms of schaffer collateral-CA1 synaptic plasticity in mice with a disruption of the CPEB-1 gene. Learn Mem 11: 318-327.

Barco A, Alarcon JM, Kandel ER. 2002. Expression of constitutively active CREB protein facilitates the late phase of long-term potentiation by enhancing synaptic capture. Cell 108: 689-703.

Belmeguenai A, Hansel C. 2005. A role for protein phosphatases $1,2 \mathrm{~A}$, and $2 \mathrm{~B}$ in cerebellar long-term potentiation. J Neurosci 25: 10768-10772.

Bougie JK, Cai D, Hastings M, Farah CA, Chen S, Fan X, McCamphill PK, Glanzman DL, Sossin WS. 2012. Serotonin-induced cleavage of the atypical protein kinase $\mathrm{C}$ Apl III in Aplysia. J Neurosci 32: 14630-14640.

Casadio A, Martin KC, Giustetto M, Zhu H, Chen M, Bartsch D, Bailey CH, Kandel ER. 1999. A transient, neuron-wide form of CREB-mediated long-term facilitation can be stabilized at specific synapses by local protein synthesis. Cell 99: 221-237.

Chen PJ, Huang YS. 2012. CPEB2-eEF2 interaction impedes HIF-1 $\alpha$ RNA translation. EMBO J 31: 959-971.

Coustou V, Deleu C, Saupe S, Begueret J. 1997. The protein product of the het-s heterokaryon incompatibility gene of the fungus Podospora anserina behaves as a prion analog. Proc Natl Acad Sci 94: 9773-9778.

Crick F. 1984. Memory and molecular turnover. Nature 312: 101.

Crow ET, Li L. 2011. Newly identified prions in budding yeast, and their possible functions. Semin Cell Dev Biol 22: $452-459$.

Drisaldi B, Colnaghi L, Fioriti L, Rao N, Myers C, Snyder AM, Metzger DJ, Tarasoff J, Konstantinov E, Fraser P, et al. 2015. SUMOylation is an inhibitory constraint that regulates the prion-like aggregation and activity of CPEB3. Cell Rep 11: 1694-1702.

Dudek SM, Fields RD. 2002. Somatic action potentials are sufficient for late-phase LTP-related cell signaling. Proc Natl Acad Sci 99: 3962-3967.

Eaglestone SS, Cox BS, Tuite MF. 1999. Translation termination efficiency can be regulated in Saccharomyces cerevisiae by environmental stress through a prion-mediated mechanism. EMBO J 18: 1974-1981.

Fioriti L, Myers C, Huang YY, Li X, Stephan J, Trifilieff P, Kosmidis S, Drisaldi B, Pavlopoulos E, Kandel ER. 2015. The persistence of hippocampal-based memory requires protein synthesis mediated by the prion-like protein CPEB3. Neuron 86: 1433-1448.

Fiumara F, Fioriti L, Kandel ER, Hendrickson WA. 2010. Essential role of coiled coils for aggregation and activity of Q/N-rich prions and PolyQ proteins. Cell 143: $1121-$ 1135 .

Fiumara F, Rajasethupathy P, Antonov I, Kosmidis S, Sossin W, Kandel E. 2015. MicroRNA-22 gates long-term heterosynaptic plasticity in Aplysia through presynaptic regulation of CPEB and downstream targets. Cell Rep 11: 1866-1875.

Frey U, Morris RG. 1997. Synaptic tagging and long-term potentiation. Nature 385: 533-536.

Frey U, Morris RG. 1998. Weak before strong: Dissociating synaptic tagging and plasticity-factor accounts of lateLTP. Neuropharmacology 37: 545-552.

Ganusova EE, Ozolins LN, Bhagat S, Newnam GP, Wegrzyn RD, Sherman MY, Chernoff YO. 2006 Modulation of prion formation, aggregation, and toxicity by the actin cytoskeleton in yeast. Mol Cell Biol 26: 617-629.

Hake LE, Richter JD. 1994. CPEB is a specificity factor that mediates cytoplasmic polyadenylation during Xenopus oocyte maturation. Cell 79: 617-627.

Halfmann R, Jarosz DF, Jones SK, Chang A, Lancaster AK, Lindquist S. 2012. Prions are a common mechanism for phenotypic inheritance in wild yeasts. Nature 482: $363-$ 368 .

Heinrich SU, Lindquist S. 2011. Protein-only mechanism induces self-perpetuating changes in the activity of neuronal Aplysia cytoplasmic polyadenylation elementbinding protein (CPEB). Proc Natl Acad Sci 108: 29993004 .

Hosoda N, Funakoshi Y, Hirasawa M, Yamagishi R, Asano Y, Miyagawa R, Ogami K, Tsujimoto M, Hoshino S. 2011. Anti-proliferative protein Tob negatively regulates CPEB3 target by recruiting Caf1 deadenylase. EMBO J 30: $1311-1323$.

Hou F, Sun L, Zheng H, Skaug B, Jiang QX, Chen ZJ. 2011. MAVS forms functional prion-like aggregates to activate and propagate antiviral innate immune response. Cell 146: $448-461$.

Huang YS, Kan MC, Lin CL, Richter JD. 2006. CPEB3 and CPEB4 in neurons: Analysis of RNA-binding specificity and translational control of AMPA receptor GluR2 mRNA. EMBO J 25: 4865-4876.

Ishimaru D, Andrade LR, Teixeira LS, Quesado PA, Maiolino LM, Lopez PM, Cordeiro Y, Costa LT, Heckl WM Weissmüller G, et al. 2003. Fibrillar aggregates of the tumor suppressor p53 core domain. Biochemistry 42: 9022-9027.

Jarosz DF, Lancaster AK, Brown JC, Lindquist S. 2014. An evolutionarily conserved prion-like element converts wild fungi from metabolic specialists to generalists. Cell 58: $1072-1082$.

Kandel ER. 2001. The molecular biology of memory storage: A dialogue between genes and synapses. Science 294: 1113-1120.

Keleman K, Krüttner S, Alenius M, Dickson BJ. 2007. Function of the Drosophila CPEB protein Orb2 in long-term courtship memory. Nat Neurosci 10: 1587-1593.

Kikuchi S, Fujimoto K, Kitagawa N, Fuchikawa T, Abe M, Oka K, Takei K, Tomita M. 2003. Kinetic simulation of signal transduction system in hippocampal long-term potentiation with dynamic modeling of protein phosphatase 2A. Neural Netw 16: 1389-1398. 
K. Si and E.R. Kandel

Krüttner S, Stepien B, Noordermeer JN, Mommaas MA Mechtler K, Dickson BJ, Keleman K. 2012. Drosophila CPEB Orb2A mediates memory independent of its RNA-binding domain. Neuron 76: 383-395.

Lee SH, Lim CS, Park H, Lee JA, Han JH, Kim H, Cheang YH, Lee SH, Lee YS, Ko HG, et al. 2007. Nuclear translocation of CAM-associated protein activates transcription for long-term facilitation in Aplysia. Cell 129: 801812.

Majumdar A, Cesario WC, White-Grindley E, Jiang H, Ren F, Khan MR, Li L, Choi EM, Kannan K, Guo F, et al. 2012. Critical role of amyloid-like oligomers of Drosophila Orb2 in the persistence of memory. Cell 148: 515-529.

Martin KC, Casadio A, Zhu H, Yaping E, Rose JC, Chen M, Bailey CH, Kandel ER. 1997a. Synapse-specific, longterm facilitation of Aplysia sensory to motor synapses: A function for local protein synthesis in memory storage. Cell 91: 927-938.

Martin KC, Michael D, Rose JC, Barad M, Casadio A, Zhu H, Kandel ER. 1997b. MAP kinase translocates into the nucleus of the presynaptic cell and is required for longterm facilitation in Aplysia. Neuron 18: 899-912.

Mastushita-Sakai T, White-Grindley E, Samuelson J, Seidel C, Si K. 2010. Drosophila Orb2 targets genes involved in neuronal growth, synapse formation, and protein turnover. Proc Natl Acad Sci 107: 11987-11992.

Meng Y, Zhang Y, Tregoubov V, Janus C, Cruz L, Jackson M, Lu WY, MacDonald JF, Wang JY, Falls DL, et al. 2002. Abnormal spine morphology and enhanced LTP in LIMK-1 knockout mice. Neuron 35: 121-133.

Miniaci MC, Kim JH, Puthanveettil SV, Si K, Zhu H, Kandel ER, Bailey CH. 2008. Sustained CPEB-dependent local protein synthesis is required to stabilize synaptic growth for persistence of long-term facilitation in Aplysia. Neuron 59: 1024-1036.

Mulkey RM, Herron CE, Malenka RC. 1993. An essential role for protein phosphatases in hippocampal long-term depression. Science 261: 1051-1055.

Pavlopoulos E, Trifilieff P, Chevaleyre V, Fioriti L, Zairis S, Pagano A, Malleret G, Kandel ER. 2011. Neuralized1 activates CPEB3: A function of non-proteolytic ubiquitin in synaptic plasticity and memory storage. Cell 147: 1369-1383.

Pi HJ, Lisman JE. 2008. Coupled phosphatase and kinase switches produce the tristability required for long-term potentiation and long-term depression. J Neurosci 28: 13132-13138.

Polymenidou M, Cleveland DW. 2012. Prion-like spread of protein aggregates in neurodegeneration. J Exp Med 209: 889-893.

Prusiner SB. 1982. Novel proteinaceous infectious particles cause scrapie. Science 216: 136-144.

Prusiner SB. 1994. Biology and genetics of prion diseases. Annu Rev Microbiol 48: 655-686.

Puthanveettil S, Kandel ER. 2011. Molecular mechanisms for the initiation and maintenance of long-term memory storage. In Two faces of evil: Cancer and neurodegeneration (ed. Curran T, Christen Y), pp. 143-160. Springer, New York.
Raveendra BL, Siemer AB, Puthanveettil SV, Hendrickson WA, Kandel ER, McDermott AE. 2013. Characterization of prion-like conformational changes of the neuronal isoform of Aplysia CPEB. Nat Struct Mol Biol 20: 495501.

Sacktor TC. 2011. How does PKMz maintain long-term memory? Nat Rev Neurosci 12: 9-15.

Schratt GM, Tuebing F, Nigh EA, Kane CG, Sabatini ME, Kiebler M, Greenberg ME. 2006. A brain-specific microRNA regulates dendritic spine development. Nature 439: 283-289.

Shorter J, Lindquist S. 2005. Prions as adaptive conduits of memory and inheritance. Nat Rev Genet 6: 435-450.

Si K, Giustetto M, Etkin A, Hsu R, Janisiewicz AM, Miniaci MC, Kim JH, Zhu H, Kandel ER. 2003a. A neuronal isoform of CPEB regulates local protein synthesis and stabilizes synapse specific long-term facilitation in Aplysia. Cell 115: 893-904.

Si K, Lindquist S, Kandel ER. 2003b. A neuronal isoform of the Aplysia CPEB has prion-like properties. Cell 115: 879-891.

Si K, Choi YB, White-Grindley E, Majumdar A, Kandel ER. 2010. Aplysia CPEB can form prion-like multimers in sensory neurons that contribute to long-term facilitation. Cell 140: 421-435.

Stephan JS, Fioriti L, Lamba N, Derkatch IL, Kandel ER. 2015. The CPEB3 protein important in memory persistence is a functional prion that interacts with the actin cytoskeleton. Cell Rep 11: 1772-1785.

Theis M, Si K, Kandel ER. 2003. Two previously undescribed members of the mouse CPEB family of genes and their inducible expression in the principal cell layers of the hippocampus. Proc Natl Acad Sci 100: 9602-9607.

Tompa P, Friedrich P. 1998. Prion proteins as memory molecules: A hypothesis. Neuroscience 86: 1037-1043.

True HL, Lindquist SL. 2000. A yeast prion provides a mechanism for genetic variation and phenotypic diversity. Nature 407: 477-483.

True HL, Berlin I, Lindquist SL. 2004 Epigenetic regulation of translation reveals hidden genetic variation to produce complex traits. Nature 431: 184-187.

White-Grindley E, Li L, Mohammad Khan R, Ren F, Saraf A, Florens L, Si K. 2014. Contribution of Orb2A stability in regulated amyloid-like oligomerization of Drosophila Orb2. PLoS Biol 12: e1001786.

Wickner RB. 1994. [URE3] as an altered URE2 protein: Evidence for a prion analog in Saccharomyces cerevisiae. Science 264: 566-569.

Yoshida Y, Tanaka S, Umemori H, Minowa O, Usui M, Ikematsu N, Hosoda E, Imamura T, Kuno J, Yamashita T, et al. 2000. Negative regulation of BMP/Smad signaling by Tob in osteoblasts. Cell 103: 1085-1097.

Yoshida Y, von Bubnoff A, Ikematsu N, Blitz IL, Tsuzuku JK, Yoshida EH, Umemori H, Miyazono K, Yamamoto T, Cho KW. 2003. Tob proteins enhance inhibitory Smadreceptor interactions to repress BMP signaling. Mech Dev 120: $629-637$. 


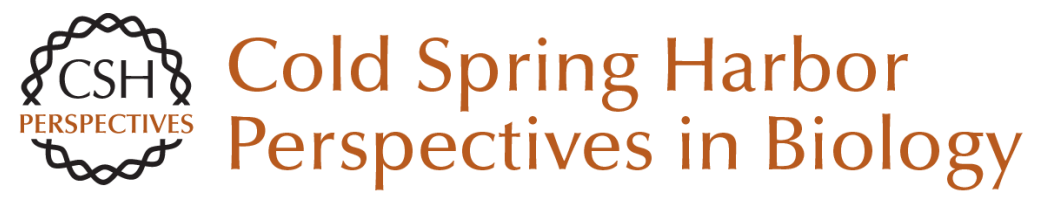

\section{The Role of Functional Prion-Like Proteins in the Persistence of Memory}

Kausik Si and Eric R. Kandel

Cold Spring Harb Perspect Biol 2016; doi: 10.1101/cshperspect.a021774

Subject Collection Learning and Memory

Large-Scale Fluorescence Calcium-Imaging

Methods for Studies of Long-Term Memory in

Behaving Mammals

Pablo Jercog, Thomas Rogerson and Mark J. Schnitzer

Exploring Memory Representations with Activity-Based Genetics Mark Mayford and Leon Reijmers

The Origins and Organization of Vertebrate

Pavlovian Conditioning Michael S. Fanselow and Kate M. Wassum

The Corticohippocampal Circuit, Synaptic Plasticity, and Memory Jayeeta Basu and Steven A. Siegelbaum

Motor Learning and the Cerebellum Chris I. De Zeeuw and Michiel M. Ten Brinke

The Striatum: Where Skills and Habits Meet Ann M. Graybiel and Scott T. Grafton

Molecular Genetic Strategies in the Study of Corticohippocampal Circuits Christopher C. Angelakos and Ted Abel

Nonassociative Learning in Invertebrates John H. Byrne and Robert D. Hawkins
The Role of Functional Prion-Like Proteins in the

Persistence of Memory

Kausik Si and Eric R. Kandel

Working Memory: Maintenance, Updating, and the Realization of Intentions

Lars Nyberg and Johan Eriksson

Memory Retrieval in Mice and Men

Aya Ben-Yakov, Yadin Dudai and Mark R. Mayford

Reconsolidation and the Dynamic Nature of Memory Karim Nader

Memory Consolidation

Larry R. Squire, Lisa Genzel, John T. Wixted, et al.

Structural Components of Synaptic Plasticity and

Memory Consolidation Craig H. Bailey, Eric R. Kandel and Kristen M. Harris

Associative Learning in Invertebrates Robert D. Hawkins and John H. Byrne

The Regulation of Transcription in Memory Consolidation Cristina M. Alberini and Eric R. Kandel

For additional articles in this collection, see http://cshperspectives.cshlp.org/cgi/collection/

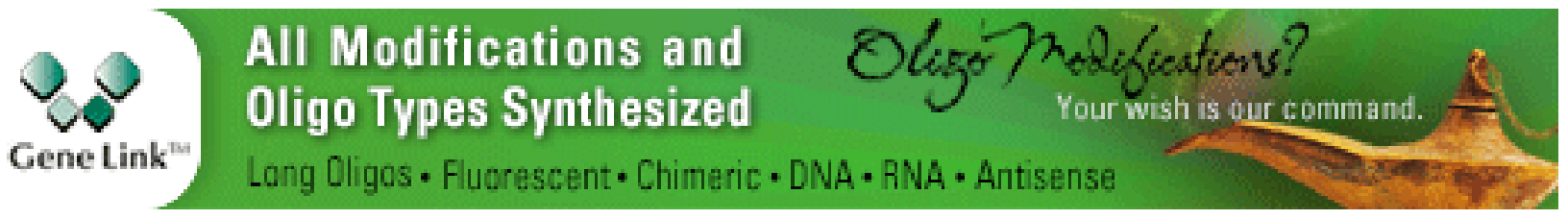

